\title{
Insulin glargine/lixisenatide in type 2 diabetes: a profile of its use
}

\author{
Emma Deeks ${ }^{1}$
}

Published online: 30 August 2019

(c) Springer Nature 2019, corrected publication 2019

\begin{abstract}
Subcutaneous insulin glargine/lixisenatide [Suliqua ${ }^{\circledR} 100 / 33$ and 100/50 (EU); Soliqua ${ }^{\circledR} 100 / 33$ (USA)] is a titratable, fixedratio combination of a long-acting basal insulin analogue + a glucagon-like peptide-1 receptor agonist (GLP-1 RA) approved to treat inadequately controlled type 2 diabetes (T2D) in adults. Once-daily insulin glargine/lixisenatide provided glycaemic control better than that of insulin glargine or lixisenatide in insulin-naive patients (when added to metformin) and better than that of insulin glargine in insulin-experienced patients (when used \pm metformin) in phase 3 trials in adults with inadequately controlled T2D. It also had a beneficial effect on bodyweight and did not increase the frequency of hypoglycaemia versus insulin glargine. Insulin glargine/lixisenatide is generally well tolerated and offers the convenience of once-daily administration of two subcutaneous antihyperglycaemic agents. It is, therefore, a valuable option for improving glycaemic control in adults with T2D when this has not been provided by metformin alone or metformin + another oral antihyperglycaemic agent or + basal insulin.
\end{abstract}

Adis evaluation of insulin glargine/lixisenatide in the treatment of T2D

Once-daily fixed-ratio combination of a long-acting insulin analogue (insulin glargine) + a GLP-1 RA (lixisenatide)

Titratable to meet the insulin needs of the individual patient

Provides better glycaemic control as an add-on to metformin than insulin glargine or lixisenatide in insulinnaive patients

Provides better glycaemic control than insulin glargine, when used \pm metformin, in insulin-experienced patients

Does not increase hypoglycaemic risk vs insulin glargine

Safety profile is generally consistent with its components

Additional information for this Adis Drug Q\&A can be found at https://doi.org/10.6084/m9.figshare.9696338.

Emma Deeks

demail@springer.com

1 Springer Nature, Private Bag 65901, Mairangi Bay, Auckland 0754, New Zealand

\section{What is the rationale for using insulin glargine/lixisenatide in type $\mathbf{2}$ diabetes?}

Type 2 diabetes (T2D) is a chronic progressive disease resulting from dysregulation of glucose homeostasis [1,2]. Maintenance of good glycaemic control [e.g. a glycated haemoglobin $\left(\mathrm{HbA}_{1 \mathrm{c}}\right)$ level of $<7 \%$ [3], although targets may be personalized $[4,5]]$ is key in preventing the long-term microvascular, and potentially also macrovascular, complications of diabetes $[5,6]$. To this end, several classes of antihyperglycaemic drugs (ADs) targeting different pathogenic pathways are available, including more recent classes such as glucagon-like peptide-1 receptor agonists (GLP-1 RAs; e.g. dulaglutide, exenatide, liraglutide, lixisenatide, semaglutide), dipeptidyl peptidase-4 (DPP-4) inhibitors (e.g. alogliptin, linagliptin, saxagliptin, sitagliptin)] and sodium glucose cotransporter-2 (SGLT2) inhibitors (e.g. canagliflozin, dapagliflozin, empagliflozin, ertugliflozin) $[2,7]$.

T2D guidelines emphasize an individualized stepwise approach to pharmacotherapy, basing treatment selection on key patient characteristics, including hypoglycaemia risk and comorbidities [e.g. cardiovascular (CV) disease, renal disease, bodyweight], as well as drug characteristics [e.g. administration route/frequency, cost, adverse event (AE) profile] [5, 7]. The AE profiles of the various $\mathrm{AD}$ classes may need consideration as they can differ. For example, some increase the risk of bodyweight gain (sulfonylureas, 
meglitinides, thiazolidinediones, insulin), whereas others have beneficial (metformin, GLP-1 RAs, SGLT2 inhibitors, $\alpha$-glucosidase inhibitors) or neutral (DPP-4 inhibitors) bodyweight effects [8]. Some also increase the risk of hypoglycaemia (sulfonylureas, insulin, meglitinides), while others are associated with gastrointestinal (GI) AEs (metformin, GLP-1 RAs, $\alpha$-glucosidase inhibitors), lactic acidosis (metformin), fracture and oedema/heart failure (thiazolidinediones), or genitourinary infections, polyuria, volume depletion/hypotension/dizziness and increased low-density lipoprotein levels (SGLT2 inhibitors) [5].

Metformin remains the first-line pharmacotherapy recommended for most patients, based on its low cost and well established efficacy/safety profile (no hypoglycaemia and potential for slight bodyweight loss and CV risk reduction) $[5,7]$. However, as the disease progresses, intensification of pharmacotherapy with at least two drugs is often required for adequate glycaemic control to be maintained [5], with several drugs also now available in convenient fixed-dose or titratable fixed-ratio combinations, which may potentially improve compliance [9]. Combination treatment with a basal insulin + a GLP-1 RA, through their complementary mechanisms of action, is one strategy for intensifying pharmacotherapy to improve glycaemic control and has fewer limiting AEs than either agent individually [10,11]. Insulin glargine/ lixisenatide [Suliqua ${ }^{\circledR} 100 / 33$ and 100/50 (EU); Soliqua ${ }^{\circledR}$ $100 / 300$ (USA)] is a fixed-ratio combination of a long-acting basal insulin analogue + a GLP-1 RA available for the treatment of adults with inadequately controlled T2D $[12,13]$.

\section{For whom is treatment with insulin glargine/ lixisenatide indicated?}

In the EU and many other countries worldwide, insulin glargine/lixisenatide (in combination with metformin) is approved to improve glycaemic control in adults with T2D when glycaemic control has not been provided by metformin alone or metformin + another oral AD (OAD) or + basal insulin [12]. In the USA, it is approved as an adjunct to diet and exercise to improve glycaemic control in adults with T2D [13].

This article focuses on the use of insulin glargine/lixisenatide in the EU (i.e. Suliqua). In this region, fixed-ratio combinations of insulin glargine/lixisenatide are available in two different strength Suliqua pens that provide different dose options, based on the dose range of the pen [12]:

- Suliqua $100 \mathrm{U} / \mathrm{mL}+50 \mu \mathrm{g} / \mathrm{mL}$ solution for injection in a prefilled pen Delivers dose steps of 10-40 units of insulin glargine $+5-20 \mu \mathrm{g}$ of lixisenatide; hereafter referred to as the Suliqua (10-40) pen.
- Suliqua $100 \mathrm{U} / \mathrm{mL}+33 \mu \mathrm{g} / \mathrm{mL}$ solution for injection in a prefilled pen Delivers dose steps of 30-60 units of insulin glargine $+10-20 \mu \mathrm{g}$ of lixisenatide; hereafter referred to as the Suliqua (30-60) pen.

The starting dose of insulin must be individualized based on the patient's insulin dose prior to initiating Suliqua, then titrated based on clinical response and the insulin needs of the patient (Table 1) [12]. The lixisenatide dose is increased or decreased along with the insulin glargine dose, with the increment depending on which pen is used. Table 1 provides a summary of the use of Suliqua pens in the EU [12], as representative of their use in countries where these pens are approved.

\section{What are the pharmacological properties of insulin glargine and lixisenatide?}

\section{Pharmacodynamic profile}

Insulin glargine and lixisenatide target different pathways in glucose homeostasis. When combined as fixed-ratio insulin glargine/lixisenatide, their complementary mechanisms of action provide sustained lowering of fasting plasma glucose (FPG; key target of insulin glargine) and postprandial plasma glucose (PPG; main target of lixisenatide) levels after all meals, thereby improving glycaemic control $[12,13]$.

- Insulin glargine Reduces blood glucose levels via inhibition of glucose production in the liver [14]. The glucoselowering effect of intravenous insulin glargine is approximately the same as that for human insulin when given at the same dose [12].

- Lixisenatide A highly selective, high affinity GLP-1 RA that acts in the same glucose-dependent manner as native GLP-1 in providing glucose homeostasis. It stimulates insulin secretion when blood glucose is increased, but not at normoglycaemia, which limits the risk of hypoglycaemia. It also suppresses glucagon secretion in a glucose-dependent manner, thereby preserving the rescue mechanism of glucagon secretion in patients experiencing hypoglycaemia, and delays gastric emptying, which reduces PPG levels by reducing the rate at which mealderived glucose is absorbed $[12,13,15]$. Its effects are exerted predominantly during the prandial period [16].

The combination of insulin glargine + lixisenatide has a more pronounced effect on glycaemic control than its individual components $[17,18]$. For example, in a study in patients with T2D receiving metformin, lixisenatide + insulin glargine had an additive effect $(p<0.05)$ on first-phase insulin secretion [17]. 
Table 1 Summary of the prescribing information of insulin glargine/lixisenatide (Suliqua 10-40 and 30-60) to improve glycaemic control in adults with type 2 diabetes when this has not been provided by metformin \pm another oral antihyperglycaemic or basal insulin in the EU [12]

How should Suliqua (10-40 or 30-60) pens be stored?

Prior to first use $\quad$ Keep refrigerated $\left(2-8{ }^{\circ} \mathrm{C}\right)$ until $1-2 \mathrm{~h}$ before use, at which point remove and store at $<25^{\circ} \mathrm{C}$

After first use Store (with cap replaced and without needle attached) at $<25^{\circ} \mathrm{C}$, but do not refrigerate or freeze

How should Suliqua be administered?

Before initiation Discontinue treatment with basal insulin and/or OADs other than metformin

Before each injection Instruct pts to check the pen label (to ensure that the Suliqua pen is being used, as opposed to another inject-

Injection frequency and timing

Injection type and site

able medication, and that the pen is of the correct strength) and to use a new needle

Once daily within $1 \mathrm{~h}$ before a meal (preferably prior to the same meal each day)

Initial dosage

Inject subcutaneously into the abdomen, deltoid or thigh, rotating sites within the same region to reduce lipodystrophy risk

Dosage titration

Base on prior AD treatment; do not exceed a lixisenatide starting dose of $>10 \mu \mathrm{g}$

Maximum daily dose $\quad$ by fasting plasma glucose)

How should Suliqua be used in special populations?

Pts with renal impairment Mild or moderate impairment: frequently monitor glucose levels and adjust dosage as necessary (insulin metabolism may be reduced)

Severe impairment or ESRD: not recommended (therapeutic experience insufficient)

Pts with hepatic impairment Frequently monitor glucose levels and adjust dosage as necessary (gluconeogenesis and insulin metabolism may be reduced)

Elderly pts (aged $\geq 65$ years) $\quad$ Adjust dosage based on glucose monitoring (renal function deterioration may reduce insulin needs)

Pts with severe GI disease Use is not recommended (Suliqua not studied in these pts; GI AEs can occur with GLP-1 RAs)

What other special warnings/precautions pertain to the use of Suliqua?

Acute pancreatitis (GLP-1 Discontinue Suliqua if acute pancreatitis is suspected and do not restart if it is confirmed

RAs increase risk)

Hypoglycaemia (most common AE with Suliqua)

Dehydration

Use with caution in pts with a history of pancreatitis

Closely monitor factors that may increase hypoglycaemia risk (e.g. inadequate food intake; concomitant use of certain drugs) and adjust the Suliqua dosage accordingly

Antibody formation Dosage adjustments may be necessary (albeit rarely) if antibodies form against Suliqua components

Do not use in pts with type 1 diabetes or as a treatment for diabetic ketoacidosis

\section{What clinically relevant drug interactions may potentially occur with Suliqua?}

Oral medications (lixisenatide delays gastric emptying, which may reduce absorption of coadministered oral drugs)

Drugs with narrow therapeutic indices or that need careful monitoring: use concomitantly with caution; advise standardized administration relative to Suliqua, and follow pts closely (particularly when Suliqua is initiated); advise administering those to be taken with food with a meal at which Suliqua is not administered

Drugs with efficacy threshold concentrations or in gastro-resistant formulations: advise taking $\geq 1 \mathrm{~h}$ prior to, or $4 \mathrm{~h}$ after, injecting Suliqua

Drugs requiring rapid GI absorption: use concomitantly with caution

Warfarin and other coumarins Frequently monitor pts when starting or stopping Suliqua in recipients of these drugs

Drugs that affect glucose metabolism ${ }^{\mathrm{b}}$

Dosage of Suliqua may need adjustment

Do not use Suliqua in combination with a sulfonylurea, due to the increased hypoglycaemia risk

$A D s$ antihyperglycaemic drugs, $A E$ adverse event, $B G$ blood glucose, ESRD end-stage renal disease, GI gastrointestinal, GLP-1 RAs glucagonlike peptide-1 receptor agonists, MAOIs monoamine oxidase inhibitors, OADs oral ADs, $p t(s)$ patient(s)

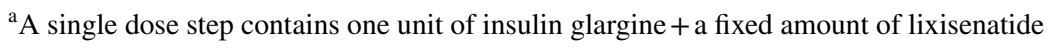

${ }^{b}$ Drugs that enhance BG lowering (e.g. ADs, ACE inhibitors, disopyramide, fibrates, fluoxetine, MAOIs, pentoxifylline, propoxyphene, salicylates, sulfonamide antibacterials), reduce BG lowering (e.g. corticosteroids, danazol, diazoxide, diuretics, glucagon, isoniazid, oestrogens, progestogens, phenothiazine derivatives, somatropin, sympathomimetic drugs, thyroid hormones, atypical antipsychotics, protease inhibitors) or potentiate/weaken BG-lowering of insulin (e.g. alcohol, $\beta$-blockers, clonidine, lithium salts)

When potential postprandial/meal-induced renal effects of lixisenatide were assessed in patients with T2D also receiving insulin glargine in an 8-week study, lixisenatide did not impact the majority of renal haemodynamic or renin-angiotensin-aldosterone system measures or any of the renal damage markers evaluated [19], although significantly ( $p<0.05$ vs baseline and/or insulin glulisine) increased postprandial/meal-induced urinary excretion of 
sodium [19, 20], chloride [20] and potassium [20], reduced postprandial excretion of calcium, magnesium and phosphate [20], and increased postprandial blood pressure [19, 21] and arterial pressure [19].

\section{Pharmacokinetic profile}

No clinically relevant differences in exposure to insulin glargine or lixisenatide were evident after subcutaneous administration of insulin glargine/lixisenatide compared with separate simultaneous injections of insulin glargine and lixisenatide in patients with type 1 diabetes [12]. Following subcutaneous administration of insulin glargine/ lixisenatide combinations, insulin glargine exhibited no pronounced peak and lixisenatide reached maximum plasma concentrations in a median of $2.5-3 \mathrm{~h}$; the apparent volume of distribution of insulin glargine and lixisenatide was $1700 \mathrm{~L}$ and $100 \mathrm{~L}$, respectively [12].

Insulin glargine is rapidly metabolized to two active metabolites (M1 and M2) in patients with diabetes, with the effects of subcutaneous insulin glargine being largely accounted for by exposure to M1 (its principal circulating metabolite) [12]. Being a peptide, elimination of lixisenatide occurs via glomerular filtration and subsequent tubular reabsorption and metabolic degradation, with the smaller peptides and amino acids produced then re-entering protein metabolism pathways. After multiple-dose administration in patients with $\mathrm{T} 2 \mathrm{D}$, the mean terminal half-life of lixisenatide was $\approx 3 \mathrm{~h}$ and the mean apparent clearance was $\approx 35 \mathrm{~L} / \mathrm{h} \mathrm{[12].}$

\section{What is the efficacy of insulin glargine/ lixisenatide?}

Two randomized, open-label, multinational, phase 3 trials (LixiLan-O [22] and LixiLan-L [23]) have evaluated the clinical efficacy of subcutaneous insulin glargine/lixisenatide in the treatment of T2D. LixiLan-O assessed its efficacy as an add-on to metformin in adults with inadequate glycaemic control despite $\geq 3$ months treatment with metformin, \pm a second OAD (57.9\% were receiving a second OAD) [22], whereas LixiLan-L assessed its efficacy in adults with inadequate glycaemic control on basal insulin + up to two OADs [most commonly metformin $(\approx 90 \%$ of patients) \pm another OAD] [23]. In both trials, all OADs other than metformin were discontinued during a prerandomization phase [22, 23]. During this phase, patients in LixiLan-O had the daily dose of metformin titrated to $\geq 2000 \mathrm{mg}$ or to the maximum tolerated [22]; patients in LixiLan-L continued or switched to insulin glargine, the daily dose of which was titrated and/or stabilized [23].
Patients whose glycaemic control remained inadequate were randomized to study drug $[22,23]$.

\section{In insulin-naive patients}

Add-on insulin glargine/lixisenatide provided glycaemic improvements superior to those of add-on insulin glargine or lixisenatide, according to pre-specified hierarchical testing of the least-square mean (LSM) change from baseline to week 30 in $\mathrm{HbA}_{1 \mathrm{c}}$ in the modified intent-to-treat population of LixiLan-O (primary endpoint; Table 2) [22]. Consistent with these findings, significantly more patients in the insulin glargine/lixisenatide group than in either comparator group achieved a target $\mathrm{HbA}_{1 \mathrm{c}}$ of $<7$ or $\leq 6.5 \%$ at week 30 (Table 2) [22].

In terms of other glycaemic parameters, the insulin glargine/lixisenatide group had a LSM change from baseline to week 30 in FPG levels that did not differ from that in the insulin glargine group (reflecting the similar basal insulin titration to target FPG levels in each group), but was more favourable than in the lixisenatide group (Table 2) [22]. At this timepoint, insulin glargine/lixisenatide also improved 2-h PPG relative to each comparator (Table 2) and 2-h PPG excursion relative to insulin glargine (LSM change -2.3 vs $-0.2 \mathrm{mmol} / \mathrm{L} ; p<0.0001$ ) in a standardized meal test. Insulin glargine/lixisenatide recipients also had a greater improvement in mean 7-point self-monitored plasma glucose (SMPG) than insulin glargine or lixisenatide recipients after 30 weeks' treatment [LSM treatment differences (TDs) were -0.69 and $-1.40 \mathrm{mmol} / \mathrm{L}$; $p<0.0001$ for each]. The insulin glargine/lixisenatide group had numerically lower mean 7-point SMPG values than either comparator group at each timepoint, with the exception of pre-breakfast (when values were similar between the insulin glargine/lixisenatide and insulin glargine groups) [22].

At 30 weeks, LSM changes from baseline in bodyweight significantly favoured add-on insulin glargine/lixisenatide over add-on insulin glargine, with a slight decrease versus a slight increase seen in the respective groups; lixisenatide recipients also experienced a reduction from baseline in bodyweight (Table 2) [22]. Significantly $(p<0.0001)$ more patients in the insulin glargine/lixisenatide than in the insulin glargine group achieved the predefined composite endpoints of $\mathrm{HbA}_{1 \mathrm{c}}$ of $<7 \%$ without bodyweight gain (43 vs $25 \%$ ) and $\mathrm{HbA}_{1 \mathrm{c}}$ of $<7 \%$ without bodyweight gain or documented symptomatic hypoglycaemia (i.e. plasma glucose $\leq 3.9 \mathrm{mmol} / \mathrm{L}$ ) [32 vs 19\%]. Rates for the respective composite endpoints in the lixisenatide group were $28 \%$ (TD vs insulin glargine/lixisenatide $15.2 \%$; 95\% CI 8.1-22.4) and $26 \%$ (TD 5.6\%; 95\% CI - 1.3 to 12.6) [these comparisons were not included in pre-specified hierarchical testing] [22]. 
Table 2 Efficacy of once-daily subcutaneous insulin glargine/lixisenatide in adults with inadequately controlled type 2 diabetes in randomized open-label, 30-week phase 3 trials

\begin{tabular}{|c|c|c|c|}
\hline \multirow[t]{2}{*}{ Outcomes } & \multicolumn{2}{|c|}{ LixiLan-O $^{\mathrm{a}}[22]$ (insulin-naive pts; + MET) } & \multirow{2}{*}{$\begin{array}{l}\text { LixiLan-L }^{\mathrm{a}}[23] \text { (insulin-experi- } \\
\text { enced pts; } \pm \text { MET) } \\
\text { iGlarLixi vs iGlar ( } 366 \text { vs } 365 \text { pts) }\end{array}$} \\
\hline & iGlarLixi vs iGlar (468 vs 466 pts) & iGlarLixi vs Lixi (468 vs 233 pts) & \\
\hline \multicolumn{4}{|l|}{ Primary endpoint } \\
\hline $\begin{array}{l}\text { LSM change from } \mathrm{BL} \text { in } \mathrm{HbA}_{1 \mathrm{c}} \\
\text { (\%) }[\mathrm{TD} ; 95 \% \mathrm{CI}]\end{array}$ & $\begin{array}{l}-1.6^{* \mathrm{~b}} \text { vs }-1.3[-0.3 ;-0.4 \text { to } \\
-0.2]\end{array}$ & $\begin{array}{l}-1.6^{*} \text { vs }-0.9[-0.8 ;-0.9 \text { to } \\
-0.7]\end{array}$ & $\begin{array}{l}-1.1 * \text { vs }-0.6[-0.5 ;-0.6 \text { to } \\
\quad-0.4]\end{array}$ \\
\hline \multicolumn{4}{|l|}{ Target $\mathrm{HbA}_{1 \mathrm{c}}$ endpoints } \\
\hline $\begin{array}{l}\% \text { of pts at target } \mathrm{HbA}_{1 \mathrm{c}}<7 \% \\
\text { [TD; } 95 \% \mathrm{CI}]\end{array}$ & $74 *$ vs $59[14.3 ; 8.4-20.3]$ & $74 *$ vs $33[40.6 ; 33.6-47.6]$ & $55^{*}$ vs $30[25.5 ; 8.9-32.1]$ \\
\hline $\begin{array}{l}\% \text { of pts at target } \mathrm{HbA}_{1 \mathrm{c}} \leq 6.5 \% \\
{[\mathrm{TD} ; 95 \% \mathrm{CI}]}\end{array}$ & $56^{*}$ vs $40[16.4 ; 10.1-22.6]$ & $56^{*}$ vs $19[36.4 ; 29.8-43.0]$ & $34 *$ vs $14[19.8 ; 13.9-25.6]$ \\
\hline \multicolumn{4}{|l|}{ Other efficacy endpoints } \\
\hline $\begin{array}{l}\text { LSM change from BL in FPG } \\
(\mathrm{mmol} / \mathrm{L})[\mathrm{TD} ; 95 \% \mathrm{CI}]\end{array}$ & $\begin{array}{l}-3.5 \text { vs }-3.3[-0.2 ;-0.4 \text { to } \\
0.04]\end{array}$ & $\begin{array}{l}-3.5^{*} \text { vs }-1.5[-2.0 ;-2.2 \text { to } \\
-1.7]\end{array}$ & -0.4 vs $-0.5[0.1 ;-0.2$ to 0.4$]$ \\
\hline $\begin{array}{l}\text { LSM change from BL in 2-h } \\
\text { PPG (mmol/L) [TD; } 95 \% \mathrm{CI}]\end{array}$ & $\begin{array}{l}-5.7 \text { vs }-3.3[-2.4 ;-2.8 \text { to } \\
-2.0]\end{array}$ & $\begin{array}{l}-5.7 \text { vs }-4.6[-1.1 ;-1.6 \text { to } \\
-0.6]\end{array}$ & $\begin{array}{l}-4.7 \text { vs }-1.4[-3.3 ;-3.9 \text { to } \\
-2.8]\end{array}$ \\
\hline $\begin{array}{l}\text { LSM change from BL in body- } \\
\text { weight }(\mathrm{kg})[\mathrm{TD} ; 95 \% \mathrm{CI}]\end{array}$ & $\begin{array}{l}-0.3 * \text { vs }+1.1[-1.4 ;-1.9 \text { to } \\
-0.9]\end{array}$ & -0.3 vs $-2.3[2.0 ; 1.4-2.6]$ & $\begin{array}{l}-0.7 * \text { vs }+0.7[-1.4 ;-1.8 \text { to } \\
-0.9]\end{array}$ \\
\hline
\end{tabular}

Results shown are at 30 weeks (study end) in the modified intent-to-treat populations. The proportions of pts at target $\mathrm{HbA}_{1 \mathrm{c}}[22,23]$ and change from BL in bodyweight with Lixi + MET [22] were not included in the prespecified hierarchical testing procedure. Across trials at BL, mean $\mathrm{HbA}_{1 \mathrm{c}}$ was $8.1 \%$, FPG was 7.3-9.9 $\mathrm{mmol} / \mathrm{L}, 2-\mathrm{h}$ PPG was $\approx 15 \mathrm{mmol} / \mathrm{L}$ and bodyweight was $87-91 \mathrm{~kg}$

$B L$ baseline, $F P G$ fasting plasma glucose, $H b A_{l c}$ glycated haemoglobin, iGlar insulin glargine, iGlarLixi titratable fixed-ratio iGlar + Lixi, Lixi lixisenatide, $L S M$ least-squares mean, $P P G$ postprandial plasma glucose, $p t s$ patients, $T D$ treatment difference

$* p<0.0001$ vs comparator

a iGlarLixi and iGlar were dose titrated (to a maximum of 60 U/day) once weekly to attain a target FPG level of 4.4-5.6 mmol/L while avoiding hypoglycaemia. In LixiLan-O, iGlarLixi was initiated at 10 dose steps (i.e. $10 \mathrm{U}$ iGlar and $5 \mu \mathrm{g} / \mathrm{mL}$ Lixi); Lixi was given as a once-daily subcutaneous dose of $10 \mu \mathrm{g} / \mathrm{mL}$ for the first 2 weeks, then as $20 \mu \mathrm{g} / \mathrm{mL}$. In LixiLan-L, iGlarLixi was initiated at 20 or 30 dose steps based on the last basal insulin dose prior to randomization at the end of the 6-week run-in period

${ }^{\mathrm{b}}$ As iGlarLixi was non-inferior to both iGlar and Lixi, superiority over Lixi and subsequently iGlar could be shown

There was no difference in the final mean daily basal insulin dose between the insulin glargine/lixisenatide and insulin glargine group at week 30 (39.8 vs $40.3 \mathrm{U}$ ), with both treatments titrated to the same target FPG level [22]. In each group, $\approx 70 \%$ of patients were receiving final daily insulin doses of $30-60 \mathrm{U}$ and $\approx 44 \%$ doses of $>40$ to $\leq 60 \mathrm{U}$, with $16 \%$ and $20 \%$ of insulin glargine/lixisenatide and insulin glargine recipients, respectively, receiving the maximum permitted dose of $60 \mathrm{U}$ [22].

\section{In insulin-experienced patients}

In patients with T2D inadequately controlled with a basal insulin + up to two OADs, as well as after dosage titration and/or stabilization of insulin glargine (generally in combination with metformin), switching insulin glargine for insulin glargine/lixisenatide was associated with significantly greater improvements in $\mathrm{HbA}_{1 \mathrm{c}}$ after 30 weeks than continuation of insulin glargine in LixiLan-L (primary endpoint; Table 2) [23]. Rates of achieving $\mathrm{HbA}_{1 \mathrm{c}}$ targets of $<7$ and $\leq 6.5 \%$ were also higher in the insulin glargine/ lixisenatide than in the insulin glargine group (Table 2) [23].

There was no TD in FPG level changes from baseline to week 30 (Table 2), reflecting the titration to target of the basal insulin [23]. However, insulin glargine/lixisenatide improved postprandial glycaemic control to a significantly greater extent than insulin glargine, based on 2-h PPG excursion values (LSM change $-3.9 \mathrm{vs}-0.5 \mathrm{mmol} / \mathrm{L} ; p<0.0001$ ) and 2-h PPG (Table 2). The 7-point SMPG profile also improved to a significantly greater extent with insulin glargine/lixisenatide than insulin glargine (LSM change -1.5 vs $-0.6 \mathrm{mmol} / \mathrm{L} ; p<0.0001$ ), with values being numerically lower in the insulin glargine/lixisenatide group at all timepoints except pre-breakfast (when they did not markedly differ) [23].

The change in bodyweight at week 30 was significantly more favourable with insulin glargine/lixisenatide than with insulin glargine (Table 2) [23]. Significantly $(p<0.0001)$ more patients in the insulin glargine/lixisenatide group attained the composites of $\mathrm{HbA}_{1 \mathrm{c}}<7 \%$ with no bodyweight gain (34 vs $13 \%$ in the insulin glargine group) or $\mathrm{HbA}_{1 \mathrm{c}}<7 \%$ 
with no bodyweight gain or documented symptomatic hypoglycaemia (20 vs 9\%) [23].

There was no difference between the insulin glargine/lixisenatide and insulin glargine groups in the final mean daily dose of basal insulin at 30 weeks (46.7 $\mathrm{U}$ in each group), with 27 and $31 \%$ of patients receiving the maximum permitted dose of $60 \mathrm{U}$ at study end [23]. The glycaemic efficacy of insulin glargine/lixisenatide was generally consistent across all final dose categories of its individual components (insulin glargine and lixisenatide) when assessed post hoc [24].

\section{Additional analyses}

The efficacy of insulin glargine/lixisenatide in insulin-naive and insulin-experienced patients with T2D was further assessed in additional analyses of LixiLan-O and LixiLan-L (exploratory $[25,26]$ or post hoc [27-37] where specified).

Glycaemic benefit was evident with insulin glargine/ lixisenatide relative to insulin glargine or lixisenatide in LixiLan-O $[25,37]$ and relative to insulin glargine in Lixilan-L [26, 28, 38], regardless of the patient's baseline $[25,26,37,38] /$ screening $[28]$ T2D duration $[25,26$, $38]$, body mass index (BMI) $[25,26]$ or $\mathrm{HbA}_{1 \mathrm{c}}$ levels [25, $26,28,37]$ (including levels $\geq 8 \%[25,26]$ or $\geq 9 \%$ [28, 37]) or whether patients had inadequate glycaemic control $\left(\mathrm{HbA}_{1 \mathrm{c}}{ }^{7-9 \%}\right)$ despite receiving two OADs at screening [37]. In addition, the glycaemic efficacy of insulin glargine/lixisenatide in elderly patients (aged $\geq 65$ years) was generally consistent with that seen in younger patients (aged $<65$ years) in these studies [27]. Similarly, patient ethnicity (Hispanic vs non-Hispanic) [31], region (North America vs rest of world) [32] and risk status (low or high, according to Healthcare Effectiveness Data and Information Set) [33] did not markedly impact the relative glycaemic efficacy of insulin glargine/lixisenatide versus insulin glargine and/or lixisenatide in LixiLan-L and LixiLan-O.

Consistent with its complimentary action on FPG and PPG, insulin glargine/lixisenatide was associated with the greatest likelihood of simultaneously achieving both FPG and PPG targets in LixiLan-O [29] and LixiLan-L [39]. Insulin glargine/lixisenatide recipients who achieved both targets had the greatest $\mathrm{HbA}_{1 \mathrm{c}}$ benefits, although $\mathrm{HbA}_{1 \mathrm{c}}$ outcomes generally favoured insulin glargine/lixisenatide over insulin glargine or lixisenatide regardless of whether patients achieved FPG and PPG targets simultaneously or achieved only one [29, 39]. Likewise consistent with its FPG and PPG effects, insulin glargine/lixisenatide significantly $(p<0.001)$ reduced the difference between bedtime and morning glucose levels [35] and the proportion of patients with discordant $\mathrm{HbA}_{1 \mathrm{c}} / \mathrm{FPG}$ levels (i.e. $\mathrm{HbA}_{1 \mathrm{c}} \geq 7 \%$ and $\mathrm{FPG}<7.8 \mathrm{mmol} / \mathrm{L}$ ) [30] relative to insulin glargine in LixiLan-L.
Insulin glargine/lixisenatide reduced glycaemic variability relative to insulin glargine or lixisenatide in LixiLan-L and LixiLan-O [34]. Among the six reported parameters [i.e. mean SMPG; standard deviation of SMPG; high blood-glucose index (HBGI); area under the SMPG curve for each patient (AUCn); mean absolute glucose; mean amplitude of glycaemic excursions], all significantly $(p \leq 0.031)$ favoured insulin glargine/lixisenatide over insulin glargine and three (mean SMPG, HBGI, AUCn) significantly $(p<0.0001)$ favoured insulin glargine/lixisenatide over lixisenatide.

Capping the daily dose of insulin glargine at $60 \mathrm{U}$ in LixiLan-L and LixiLan-O (when it may exceed this in a real-world setting) is unlikely to have impacted the relative efficacy of insulin glargine/lixisenatide versus insulin glargine in these studies, according to a simulation analysis [36], with these findings supporting the hypothesis that insulin glargine/lixisenatide has glycaemic benefits beyond those of insulin glargine.

\section{What is the tolerability profile of insulin glargine/lixisenatide?}

Insulin glargine/lixisenatide was generally well tolerated during 30 weeks of therapy in LixiLan-O [22] and LixiLanL [23], with a safety profile generally consistent with those established for its individual components [22, 23]; pooled data from the trials [40] supported their individual findings.

In these studies, treatment-emergent AEs (TEAEs) occurred in around half of insulin glargine/lixisenatide and insulin glargine recipients $[22,23]$ and a majority of lixisenatide recipients [22] (Table 3 ), and were generally mild to moderate in severity. Across groups, TEAEs were not often the cause of treatment discontinuation (at least among insulin glargine/lixisenatide and insulin glargine recipients) and were serious in $4-6 \%$ of patients (Table 3) [22, 23]. Fewer than $1 \%$ of patients died due to TEAEs in any trial group (Table 3) [22, 23] and, where specified, none of the deaths were considered to be treatment related [22]. In LixiLan-L, the deaths were due to pneumonia in the insulin glargine/ lixisenatide group and gallbladder cancer or cardiopulmonary failure in the insulin glargine group [23]. There were no clinical safety concerns regarding laboratory parameters (including amylase and lipase), vital signs, physical examination or ECG parameters and no adjudicated pancreatitis AEs $[22,23]$ in either trial; one patient (in the insulin glargine group) had a pancreatic neoplasm in LixiLan-O [22].

Consistent with its individual components, hypoglycaemia is the only adverse reaction reported in the EU summary of product characteristics as being very common (i.e. incidence $\geq 10 \%$ ) with insulin glargine/lixisenatide, while common (i.e. incidence $\geq 1$ to $<10 \%$ ) adverse reactions 
associated with its use are mainly GI in nature (nausea, diarrhoea, vomiting and dizziness) [12]. Indeed, GI disorders $[22,23]$ and/or infections/infestations [23] were the most common classes of AEs to occur with insulin glargine/lixisenatide in LixiLan-O [22] or LixiLan-L [23] (Table 3).

\section{Adverse events of special interest}

\section{Gastrointestinal disorders}

In both LixiLan-O [22] and LixiLan-L [23], numerically more insulin-glargine/lixisenatide than insulin glargine recipients experienced GI disorders (Table 3), including nausea (9.6 vs 3.6\% [22]; 10.4 vs $0.5 \%$ [23]), vomiting (3.2 vs $1.5 \%$ [22]; 3.6 vs $0.5 \%$ [23]) and diarrhoea (9.0 vs $4.3 \%$ [22]; 4.4 vs $2.7 \%$ [23]). In LixiLan-O [22], each of these groups had a numerically lower incidence of GI disorders than the lixisenatide group (Table 3 ), reflecting the numerically higher rates of nausea $(24.0 \%)$ and vomiting $(6.4 \%)$ with lixisenatide than with both insulin glargine/lixisenatide and insulin glargine, and the numerically higher rate of diarrhoea $(9.0 \%)$ with lixisenatide than with insulin glargine. However, few patients discontinued study treatment because of these GI AEs across the treatment groups of either trial $(\leq 2.6 \%[22] ; \leq 1.1 \%[23])$.

GI AEs tended to occur early in the course of insulin glargine/lixisenatide (and likewise lixisenatide) therapy, with few recipients experiencing them beyond 8-9 weeks in a post hoc analysis [41] of LixiLan-O and -L. The median duration of nausea, vomiting or diarrhoea was 1-6 days with insulin glargine/lixisenatide versus 1-3 days with insulin glargine and 3-7.5 days with lixisenatide [41].

\section{Hypoglycaemic events}

In LixiLan-O [22] and LixiLan-L [23], the hypoglycaemia profile of insulin glargine/lixisenatide was generally similar to that of insulin glargine in terms of documented symptomatic events accompanied by plasma glucose levels $\leq 3.9 \mathrm{mmol} / \mathrm{L}$ (Table 3 ). The incidence of these events was approximately four times greater with these regimens than with lixisenatide in LixiLan-O [22] (as is to be expected when comparing an insulin-based therapy with a GLP-1 RA), although severe symptomatic hypoglycaemic events were uncommon regardless of treatment group across the trials (Table 3) [22, 23].

\section{Cardiovascular events}

No dedicated CV outcome trial was conducted for insulin glargine/lixisenatide [12], as the CV safety profiles of insulin glargine and lixisenatide had already been evaluated in large randomized, multinational studies: ORIGIN (an open-label trial in 12,537 adults with CV risk factors + impaired fasting glucose, impaired glucose tolerance or T2D) [42] and ELIXA (a double-blind trial in 6068 patients with T2D who had recently had an acute coronary event) [43].

After a median follow-up of 6.2 years in ORIGIN, the incidence of major adverse $\mathrm{CV}$ events (MACE) did not differ between insulin glargine and standard care [2.94 vs 2.85 events/100 PY; hazard ratio (HR) 1.02; 95\% CI 0.94-1.11] (primary outcome), with MACE being a composite of the first occurrence of $\mathrm{CV}$ death, nonfatal myocardial infarction (MI) and nonfatal stroke [42]. After a median follow-up of 25 months in combination with standard care in ELIXA, lixisenatide was noninferior, but not superior, to placebo

\section{Table 3 Tolerability profile of insulin glargine/lixisenatide in adults with type 2 diabetes in phase 3 trials}

\begin{tabular}{|c|c|c|c|c|c|c|c|c|}
\hline \multirow[t]{3}{*}{ Regimen (no. of pts) } & \multicolumn{6}{|c|}{ Treatment-emergent adverse events ( $\%$ of pts) } & \multicolumn{2}{|c|}{$\begin{array}{l}\text { Symptomatic hypoglycaemia } \\
\text { events ( } \% \text { of pts) [per patient- } \\
\text { year] }\end{array}$} \\
\hline & \multirow[t]{2}{*}{ Any } & \multirow[t]{2}{*}{ Serious } & \multicolumn{2}{|c|}{ Leading to: } & \multicolumn{2}{|c|}{ Most common class } & \multirow[t]{2}{*}{ Documented $^{\mathrm{a}}$} & \multirow[t]{2}{*}{ Severe ${ }^{\mathrm{b}}$} \\
\hline & & & $\mathrm{DC}$ & death & GI & Infect/Infest & & \\
\hline \multicolumn{9}{|c|}{ LixiLan-O (insulin-naive pts; + metformin) [22] } \\
\hline iGlarLixi + metformin (469) & 56.9 & 3.8 & 2.6 & 0.4 & 21.7 & & $25.6[1.4]$ & $0[0]$ \\
\hline iGlar + metformin $(467)$ & 48.6 & 4.1 & 1.9 & 0.6 & 12.6 & & $23.6[1.2]$ & $0.2[<0.01]$ \\
\hline Lixi + metformin (233) & 67.4 & 3.9 & 9.0 & 0.4 & 36.9 & & $6.4[0.3]$ & $0[0]$ \\
\hline \multicolumn{9}{|c|}{ LixiLan-L (insulin-experienced pts; \pm metformin) [23] } \\
\hline iGlarLixi \pm metformin $(365)$ & 53.4 & 5.5 & 2.7 & 0.3 & 17.0 & 26.8 & $40.0[3.0]$ & $1.1[0.02]$ \\
\hline iGlar \pm metformin $(365)$ & 52.3 & 4.9 & 0.8 & 0.5 & 7.9 & 30.7 & $42.5[4.2]$ & $0.3[<0.01]$ \\
\hline
\end{tabular}

DC discontinuation, GI gastrointestinal disorders, Infect/Infest infections/infestations, pts patients

${ }^{\text {a }}$ Typical hypoglycaemia symptoms accompanied by plasma glucose levels $\leq 3.9 \mathrm{mmol} / \mathrm{L}$

${ }^{\mathrm{b}}$ Requiring another person's assistance to administer carbohydrate, glucagon or other resuscitative actions 
in terms of the primary composite 4-point MACE outcome of time to first occurrence of CV death, nonfatal MI, nonfatal stroke or hospitalization for unstable angina (composite occurred in 406 vs 399 patients; HR 1.02; 95\% CI $0.89-1.17)$ [43].

The LixiLan trials were not powered to evaluate CV outcomes, although the MACE incidence was low and similar between the treatment groups of LixiLan-L [23], and few patients had adjudicated CV events with insulin glargine/ lixisenatide, insulin glargine or lixisenatide in LixiLan-O (two, seven and two patients, respectively) [22].

\section{Other}

Antibodies may form against the components of insulin glargine/lixisenatide. Among patients with T2D who received insulin glargine/lixisenatide for 30 weeks in either of the two phase 3 trials, anti-insulin glargine antibodies developed in 21.0 and $26.2 \%$ of recipients and anti-lixisenatide antibodies developed in $\approx 43 \%$ of recipients [12]. Anti-insulin glargine antibodies showed cross-reactivity to human insulin in $\approx 93 \%$ of patients. The presence of these antibodies had no clinically relevant impact on safety or efficacy [12].

Across LixiLan-O and -L, allergic reactions (urticaria) considered possibly related to insulin glargine/lixisenatide occurred in $0.3 \%$ of patients [12]. Cases of generalized allergic reaction, including anaphylactic reactions and angioedema, have occurred during insulin glargine and lixisenatide use. Few patients (1.7\%) taking insulin glargine/ lixisenatide or other insulin-containing therapies have experienced erythema, local oedema and pruritus at the injection site [12].

There have been no identified cases of thyroid carcinoma among insulin glargine/lixisenatide recipients in clinical trials or post-marketing surveillance to date [40].

\section{What is the current clinical position of insulin glargine/lixisenatide?}

Insulin glargine/lixisenatide is one of two fixed-ratio combinations of a basal insulin and GLP-1 RA currently available for subcutaneous injection in the EU [44]. Pivotal phase 3 trials indicate that once-daily insulin glargine/lixisenatide is an effective and generally well tolerated treatment option for use in addition to metformin in insulin-naive adults with T2D inadequately controlled with metformin (alone or + another OAD) and \pm metformin in insulin-experienced adults whose T2D is inadequately controlled with basal insulin ( \pm OADs). According to these studies, using insulin glargine/lixisenatide in these settings improves overall glycaemic control versus the use of insulin glargine (in insulin-naive or -experienced patients) or lixisenatide (in insulin-naive patients), as well as having a beneficial effect on bodyweight and no increase in hypoglycaemia risk versus insulin glargine.

The tolerability profile of insulin glargine/lixisenatide is generally consistent with its components. Although GI AEs may occur more frequently with insulin glargine/lixisenatide than with insulin glargine (consistent with the GI impact of lixisenatide [7]), they appear to be less common with insulin glargine/lixisenatide than with lixisenatide in the first few weeks of treatment, possibly due to slower lixisenatide titration when part of insulin glargine/lixisenatide [40].

The CV safety of ADs is also important to determine [45], with the EMA recommending a long-term, controlled outcome study with 18-24 months of follow-up for ADs suspected to have an adverse CV effect [46]. A dedicated CV outcome trial was not necessary for insulin glargine/lixisenatide, as such studies conducted separately with each of its components (+ standard care) identified no safety concerns. However, some GLP-1 RAs (liraglutide, semaglutide, dulaglutide) and SGLT2 inhibitors (empagliflozin, canagliflozin) have demonstrated CV benefit in patients with T2D. These findings are reflected in the most recent treatment guidelines of the ADA/EASD [5] and ADA [7], which recommend selecting $\mathrm{AD}$ regimens (beyond first-line metformin) on the basis of the following key factors:

- presence of atherosclerotic CV disease (use a GLP-1 RA or SGLT2 inhibitor with proven CV disease benefit);

- presence of heart failure or chronic kidney disease (use a SGLT2 inhibitor proven to reduce progression of these comorbidities is preferred or GLP-1 RA with proven CV disease benefit);

- need to minimize weight gain/promote weight loss (use a GLP-1 RA with good weight loss or SGLT2 inhibitor);

- need to minimize hypoglycaemia (use a GLP-1 RA, SGLT2 inhibitor, DPP-4 inhibitor or thiazolidinedione);

- cost, when a major issue (use a sulfonylurea or thiazolidinedione).

Addition of a basal insulin is commonly recommended in subsequent-line therapies to achieve/maintain glycaemic targets, with insulin glargine and insulin degludec (both of which have established CV safety [44]) being preferred when there is comorbid atherosclerotic CV disease, heart failure or chronic kidney disease [5, 7]. Combination regimens containing a basal insulin + a GLP-1 RA are highly effective [5], and are the first injectable combinations recommended for patients intensifying from a dual/triple oral regimen to injectable therapy $[5,7]$. In this setting, basal insulin should usually be added to the GLP-1 RA, although combined use of these agents can be considered as an initial injectable therapy in some patients (namely those with $\mathrm{HbA}_{1 \mathrm{c}}>10 \%$ and/or $>2 \%$ greater than target) $[5,7]$. 
For patients requiring basal insulin + a GLP-1 RA, guidelines recommend considering one of the approved fixed-ratio combinations (i.e. insulin glargine/lixisenatide or insulin degludec/liraglutide), as administration requires fewer injections (one daily [47]) than if the components are administered separately $[5,7]$. The fixed-ratio combinations have not yet been directly compared, although such studies would be of interest. Indirect comparisons are available [48, 49], but, given their nature, caution is required when interpreting their findings (i.e. $\mathrm{HbA}_{1 \mathrm{c}}$ and bodyweight benefits are similar between the two combinations [48] or are more favourable with insulin degludec/liraglutide [49]).

The long-term cost-effectiveness of insulin degludec/ liraglutide versus insulin glargine/lixisenatide in patients with T2D poorly controlled with basal insulin in the EU has been evaluated in pharmacoeconomic analyses from the healthcare payer perspectives of Italy [50], Denmark [51] and the Czech Republic [52] (year of costing 2018 in all analyses). They suggest that, over patient lifetimes, insulin degludec/liraglutide is cost-effective relative to insulin glargine/lixisenatide, with insulin degludec/liraglutide being more costly, but providing greater gains in quality-adjusted life-years (QALYs); the incremental costs per QALY gained were EUR7386 [50], DKK182,451 [51] and CZK695,998 or CZK348,323 (depending on whether the insulin glargine/ lixisenatide pen contained 33 or $50 \mu \mathrm{g} / \mathrm{mL}$ of lixisenatide) [52]. Further EU cost-effectiveness analyses for insulin glargine/lixisenatide would be beneficial (given the considerable societal and healthcare payer costs associated with T2D), as would longer-term clinical experience. Real-world data for insulin glargine/lixisenatide in T2D, although currently limited to a small retrospective analysis from an ambulatory care endocrinology practice $(n=23$ evaluable; $\approx 4$ months' treatment with the combination on average) [53], are supportive of clinical trial findings.

Regardless of any potential differences between the available fixed-ratio basal insulin/GLP-1 RA combinations, meta-analyses indicate that regimens combining use of these agents provide robust glycaemic control $[11,54]$, while mitigating the key drawbacks of insulins (i.e. bodyweight gain and hypoglycaemia) [11, 54] and GLP-1 RAs (GI adverse events) [54]; this may improve adherence [55] and allow patients who are concerned about such issues (but require therapy intensification) to be more accepting of taking these agents [47]. However, initiating treatment early with fixedratio insulin glargine/lixisenatide may provide better efficacy and GI tolerability than using the agents sequentially, according to indirect comparative analyses of data from LixiLan-O and - $\mathrm{L}$ versus trials that assessed add-on lixisenatide in patients with uncontrolled T2D despite initiating or intensifying insulin glargine therapy (GetGoal Duo-1 and -2) [56]; direct comparisons are required to confirm these findings.
Although not suitable for some patients (e.g. those who require a higher insulin dose or different basal insulin: GLP-1 RA ratio than is available), the fixed-ratio combinations are likely to be appropriate for most $(\approx 80 \%$ of patients) $[44,47]$. The fixed-ratio combinations may be an acceptable alternative to insulin intensification, particularly for patients who do not wish to add prandial bolus insulin because of the extra injections required [47]. Injection fears and/or a need for multiple injections are common reasons for patients to resist advancing treatment, although fixed-ratio combinations (being taken once-daily via a pen device) could potentially reduce such resistance [44]. Because of their lower propensity for hypoglycaemia, the fixed-ratio combinations may also require less frequent blood glucose monitoring than basal-bolus insulins [44], which may help to reduce patient burden.

Acknowledgements The manuscript was updated from Drugs 2017;17(12):1353-62 [15], and was reviewed by: S.C. Bain, Swansea University Medical School, Swansea, UK; G. Dimitriadis, 2nd Department of Internal Medicine, Research Institute and Diabetes Center, Athens University Medical School, Attikon University Hospital, Haidari, Greece; T. Tzotzas, St Luke's General Hospital, Thessaloniki, Greece. During the peer review process, Sanofi-Aventis, the marketingauthorization holder of insulin glargine/lixisenatide, was also offered an opportunity to provide a scientific accuracy review of their data. Changes resulting from comments received were made on the basis of scientific and editorial merit.

\section{Compliance with ethical standards}

Funding The preparation of this review was not supported by any external funding.

Conflicts of interest Emma Deeks is an employee of Adis International $\mathrm{Ltd} /$ Springer Nature, is responsible for the article content and declares no conflicts of interest.

Open Access This article is distributed under the terms of the Creative Commons Attribution-NonCommercial 4.0 International License (http://creativecommons.org/licenses/by-nc/4.0/), which permits any noncommercial use, duplication, adaptation, distribution and reproduction in any medium or format, as long as you give appropriate credit original author(s) and the source, provide a link to the Creative Commons licence and indicate if changes were made.

\section{References}

1. American Diabetes Association. 2. Classification and diagnosis of diabetes: standards of medical care in diabetes-2018. Diabetes Care. 2019;41(Suppl 1):S13-27.

2. Inzucchi SE, Bergenstal RM, Buse JB, et al. Management of hyperglycaemia in type 2 diabetes, 2015: a patient-centered approach. Update to position statement of the American Diabetes Association and the European Association for the Study of Diabetes. Diabetes Care. 2015;38:140-9.

3. American Diabetes Association. 6: Glycemic targets: standards of medical care in diabetes - 2019. Diabetes Care. 2019;42(Suppl 1):S61-70. 
4. Riddle MC, Gerstein HC, Holman RR, et al. A1C targets should be personalized to maximize benefits while limiting risks. Diabetes Care. 2018;41(6):1121-4.

5. Davies MJ, D'Alessio DA, Fradkin J, et al. Management of hyperglycemia in type 2 diabetes, 2018. A consensus report by the American Diabetes Association (ADA) and the European Association for the Study of Diabetes (EASD). Diabetes Care. 2018;41(12):2669-701.

6. Milligan S. Combination therapy for improvement of long-term macrovascular and microvascular outcomes in type 2 diabetes: rationale and evidence for early initiation. J Diabetes Complications. 2016;30:1177-85.

7. American Diabetes Association. 9. Pharmacological approaches to glycemic treatment: standards of medical care in diabetes 2019. Diabetes Care. 2019;42(Suppl 1):S90-102.

8. Apovian CM, Okemah J, O’Neil PM. Body weight considerations in the management of type 2 diabetes. Adv Ther. 2019;36(1):44-58.

9. Levin PA. Practical combination therapy based on pathophysiology of type 2 diabetes. Diabetes Metab Syndr Obes. 2016;9:355-69.

10. Wilding JPH, Bain SC. Role of incretin-based therapies and sodium-glucose co-transporter- 2 inhibitors as adjuncts to insulin therapy in type 2 diabetes, with special reference to IDegLira. Diabet Med. 2016;33(7):864-76.

11. Eng C, Kramer CK, Zinman B, et al. Glucagon-like peptide-1 receptor agonist and basal insulin combination treatment for the management of type 2 diabetes: a systematic review and metaanalysis. Lancet. 2014;384:2228-34.

12. Suliqua (insulin glargine 100 units $/ \mathrm{mL}+$ lixisenatide 33 or 50 micrograms $/ \mathrm{mL}$ ) solution in a prefilled pen: summary of product characteristics. Paris: Sanofi-Aventis Groupe; 2019.

13. Soliqua 100/33 (insulin glargine and lixisenatide injection), for subcutaneous use: US prescribing information. Bridgewater (NJ): sanofi-aventis US LLC; 2019.

14. Porcellati F, Lucidi P, Cioli P, et al. Pharmacokinetics and pharmacodynamics of insulin glargine given in the evening as compared with in the morning in type 2 diabetes. Diabetes Care. 2015;38(3):503-12.

15. Scott LJ. Insulin glargine/lixisenatide: a review in type 2 diabetes. Drugs. 2017;77(12):1353-62.

16. Vidal J. Lixisenatide: a new glucagon-like peptide 1 receptor agonists in the treatment of type 2 diabetes. Eur Endocrinol. 2013;9(2):76-81.

17. Meier JJ, Menge B, Schenker N, et al. Mechanisms of action of the glucose-lowering effect of lixisenatide in combination with insulin glargine [abstract no. 281-OR]. Diabetes. 2015;64(Suppl 1):A74.

18. Kovatchev B, Umpierrez G, Renard E. The differential and combined action of insulin glargine and lixisenatide on the fasting and post-prandial components of glucose control [abstract no. 3]. Diabetologia. 2016;59(Suppl 1):S2.

19. Tonneijck L, Muskiet MHA, Smits MM, et al. Postprandial renal haemodynamic effect of lixisenatide vs once-daily insulinglulisine in patients with type 2 diabetes on insulin-glargine: an 8-week, randomised, open-label trial. Diabetes Obes Metab. 2017;19(12):1669-80

20. Tonneijck L, Muskiet MHA, Blijdorp CJ, et al. Renal tubular effects of prolonged therapy with the GLP-1 receptor agonist lixisenatide in patients with type 2 diabetes mellitus. Am J Physiol Renal Physiol. 2019;316(2):F231-40.

21. Tonneijck L, Muskiet MHA, Twisk JW, et al. Lixisenatide versus insulin glulisine on fasting and postbreakfast systemic hemodynamics in type 2 diabetes mellitus patients. Hypertension. 2018;72(2):314-22.
22. Rosenstock J, Aronson R, Grunberger G, et al. Benefits of LixiLan, a titratable fixed-ratio combination of insulin glargine plus lixisenatide, versus insulin glargine and lixisenatide monocomponents in type 2 diabetes inadequately controlled on oral agents: the LixiLan-O randomized trial. Diabetes Care. 2016;39(11):2026-35.

23. Aroda VR, Rosenstock J, Wysham C, et al. Efficacy and safety of LixiLan, a titratable fixed-ratio combination of insulin glargine plus lixisenatide in type 2 diabetes inadequately controlled on basal insulin and metformin: the LixiLan-L randomized trial. Diabetes Care. 2016;39(11):1972-80.

24. Ritzel R, Vidal J, Aroda VR, et al. Efficacy and safety across the final dose ranges in patients with T2DM receiving insulin glargine/lixisenatide fixed-ratio combination in the LixiLan-L trial [abstract no. 1024-P]. Diabetes. 2016;65(Suppl 1):A266-7.

25. Davis M, Leiter LA, Grunberger G, et al. Impact of baseline $\mathrm{HbA}_{1 \mathrm{c}}, \mathrm{BMI}$, and diabetes duration on the efficacy and safety of LixiLan (insulin glargine/lixisenatide titratable fixed-ratio combination) vs. insulin glargine and lixisenatide in the LixiLan-O trial [abstract no. 1028-P]. Diabetes. 2016;65(Suppl 1):A268.

26. Wysham C, Bonadonna RC, Aroda VR, et al. Consistent findings in glycaemic control, body weight and hypoglycaemia with iGlarLixi (insulin glargine/lixisenatide titratable fixed-ratio combination) vs insulin glargine across baseline $\mathrm{HbA1c}$, BMI and diabetes duration categories in the LixiLan-L trial. Diabetes Obes Metab. 2017;19(10):1408-15.

27. Handelsman Y, Chovanes C, Dex T, et al. Efficacy and safety of insulin glargine/lixisenatide fixed-ratio combination in elderly patients with T2D [abstract no. 954-P]. Diabetes. 2016;65(Suppl 1):A246.

28. Niemoeller E, Souhami E, Wu Y, et al. iGlarLixi reduces glycated hemoglobin to a greater extent than basal insulin regardless of levels at screening: post hoc analysis of LixiLan-L. Diabetes Ther. 2018;9(1):373-82.

29. Desouza C, Fonseca V, Frias JP, et al. Glycemic target attainment in insulin-naive patients with T2D receiving iglarlixi [abstract no. 1093-P]. Diabetes. 2018;67(Suppl 1):A291.

30. Giorgino F, Retnakaran R, Vidal J, et al. iGlarlixi effectively reduces residual hyperglycemia in patients with type 2 diabetes on basal insulin-a post-hoc analysis from the LixiLan-1 study [abstract no. 1095-P]. Diabetes. 2018;67(Suppl 1):A292.

31. Mora P, Chao J, Saremi A, et al. Relationship of ethnicity to clinical outcomes in iglarlixi-treated patients with type 2 diabetes [abstract no. 1072-P]. Diabetes. 2018;67(Suppl 1):A284.

32. Dailey G, Bajaj HS, Dex T, et al. Post hoc efficacy and safety analysis of insulin glargine/lixisenatide fixed-ratio combination in North American patients compared with the rest of world. BMJ Open Diabetes Res Care. 2019;7(1).

33. Sugimoto DH, Dex T, Stager W, et al. Efficacy of iGlarLixi, a fixed-ratio combination of insulin glargine and lixisenatide, in patients with type 2 diabetes stratified as at high or low risk according to HEDIS measurements. Diabetes Obes Metab. 2018;20(11):2680-4.

34. Aronson R, Umpierrez G, Stager W, et al. Insulin glargine/lixisenatide fixed-ratio combination improves glycaemic variability and control without increasing hypoglycaemia. Diabetes Obes Metab. 2019;21(3):726-31.

35. Zisman A, Dex T, Roberts M, et al. Bedtime-to-morning glucose difference and iGlarLixi in type 2 diabetes: post hoc analysis of LixiLan-L. Diabetes Ther. 2018;9(5):2155-62.

36. Schmider W, Belder R, Lee M, et al. Impact of dose capping in insulin glargine/lixisenatide fixed-ratio combination trials in patients with type 2 diabetes. Curr Med Res Opin. 2019;35(6):1081-9.

37. Davies MJ, Russell-Jones D, Barber TM, et al. Glycaemic benefit of iGlarLixi in insulin-naive type 2 diabetes patients with high 
HbA1c or those with inadequate glycaemic control on two oral antihyperglycaemic drugs in the LixiLan-O randomized trial. Diabetes Obes Metab. 2019;21(8):1967-72.

38. Blonde L, Berard L, Saremi A, et al. Impact of type 2 diabetes (T2D) duration on response to iglarlixi vs. Iglar:a subanalysis of LixiLan-L [abstract no. 1094-P]. Diabetes. 2018;67(Suppl 1):A291-2.

39. Leiter LA, Chao J, Saremi A, et al. A1c target attainment in patients with T2D receiving iGlarLixi who reach PPG and FPG targets in the Lixilan-L trial [abstract no. 1089-P]. Diabetes. 2018;67(Suppl 1):A290.

40. Frias JP, Dex T, Roberts M, et al. A review of the safety and adverse event profile of the fixed-ratio combination of insulin glargine and lixisenatide. Diabetes Ther. 2019;10(1):21-33.

41. Trujillo JM, Roberts M, Dex T, et al. Low incidence of gastrointestinal adverse events over time with a fixed-ratio combination of insulin glargine and lixisenatide versus lixisenatide alone. Diabetes Obes Metab. 2018;20(11):2690-4.

42. ORIGIN Trial Investigators. Basal insulin and cardiovascular and other outcomes in dysglycemia. N Engl J Med. 2012;367(4):319-28.

43. Pfeffer MA, Claggett B, Diaz R, et al. Lixisenatide in patients with type 2 diabetes and acute coronary syndrome. N Engl J Med. 2015;373(23):2247-57.

44. Perreault L, Rodbard H, Valentine V, et al. Optimizing fixedratio combination therapy in type 2 diabetes. Adv Ther. 2019;36(2):265-77.

45. Zannad F, Stough WG, Lipicky RJ, et al. Assessment of cardiovascular risk of new drugs for the treatment of diabetes mellitus: risk assessment vs. risk aversion. Eur Heart J. 2016;2:200-5.

46. Committee for Medicinal Products for Human Use (CHMP). Guidelines on clinical investigation of medicinal products in the treatment or prevention of diabetes mellitus: revision 1. London: European Medicines Agency; 2012.

47. Blumer I, Pettus JH, Santos Cavaiola T. Fixed-ratio combination therapy for type 2 diabetes: the top ten things you should know about insulin and glucagon-like peptide-1 receptor agonist combinations. Postgrad Med. 2018;130(4):375-80

48. Cai X, Gao X, Yang W, et al. Comparison between insulin degludec/liraglutide treatment and insulin glargine/lixisenatide treatment in type 2 diabetes: a systematic review and meta-analysis. Expert Opin Pharmacother. 2017;18(17):1789-98.

49. Evans M, Billings LK, Hakan-Bloch J, et al. An indirect treatment comparison of the efficacy of insulin degludec/liraglutide (IDegLira) and insulin glargine/lixisenatide (iGlarLixi) in patients with type 2 diabetes uncontrolled on basal insulin. J Med Econ. 2018;21(4):340-7.

50. Montagnoli R, Lastoria G, Parekh W, et al. Long-term costeffectiveness analysis of IDegLira versus iGlarLixi for the treatment of patients with poorly controlled type 2 diabetes on basal insulin in the Italian setting [abstract no. PDB77]. Value Health. 2018;21(Suppl 3):S131.

51. Hvid C, Parekh W, Pohlmann J, et al. Cost-effectiveness of treating patients with type 2 diabetes poorly controlled on basal insulin with fixed-ratio combinations of basal insulin and Glp-1 receptor agonists in Denmark: IDegLira versus iGlarLixi [abstract no. PDB75 + poster]. Value Health. 2018;21(Suppl 3):S131.

52. Pohlmann J, Russel-Szymczyk M, Holik P, et al. Treating patients with type 2 diabetes mellitus uncontrolled on basal insulin in the Czech Republic: cost-effectiveness of IDegLira versus iGlarLixi. Diabetes Ther. 2019;10(2):493-508.

53. Stryker MD, Blow CA, Friedman EB, et al. Assessing the realworld use of combination insulin glargine-lixisenatide in patients with type 2 diabetes mellitus:a retrospective review from an ambulatory care endocrinology practice [abstract no. 2290-PUB]. Diabetes. 2018;67(Suppl 1):A598.

54. Liakopoulou P, Liakos A, Vasilakou D, et al. Fixed ratio combinations of glucagon like peptide 1 receptor agonists with basal insulin: a systematic review and meta-analysis. Endocrine. 2017;56(3):485-94.

55. Skolnik N, Hinnen D, Kiriakov Y, et al. Initiating titratable fixedratio combinations of basal insulin analogs and glucagon-like peptide-1 receptor agonists: what you need to know. Clin Diabetes. 2018;36(2):174-82.

56. Rosenstock J, Handelsman Y, Vidal J, et al. Propensity-scorematched comparative analyses of simultaneously administered fixed-ratio insulin glargine $100 \mathrm{U}$ and lixisenatide (iGlarLixi) vs sequential administration of insulin glargine and lixisenatide in uncontrolled type 2 diabetes. Diabetes Obes Metab. 2018;20(12):2821-9. 